Florida International University FIU Digital Commons

$10-30-2012$

\title{
Predicting Explorative Behavior by Level of Emotional Reactivity in Bobwhite Quail Neonates (Colinus virginianus)
}

Michael Suarez

Florida International University, msuar022@fiu.edu

DOI: $10.25148 /$ etd.FI13040105

Follow this and additional works at: https://digitalcommons.fiu.edu/etd

\section{Recommended Citation}

Suarez, Michael, "Predicting Explorative Behavior by Level of Emotional Reactivity in Bobwhite Quail Neonates (Colinus virginianus)" (2012). FIU Electronic Theses and Dissertations. 810.

https://digitalcommons.fiu.edu/etd/810 


\title{
FLORIDA INTERNATIONAL UNIVERSITY \\ Miami, Florida
}

\section{PREDICTING EXPLORATIVE BEHAVIOR BY LEVEL OF EMOTIONAL REACTIVITY IN BOBWHITE QUAIL NEONATES (Colinus virginianus)}

\author{
A thesis submitted in partial fulfillment of the \\ requirements for the degree of \\ MASTER OF SCIENCE \\ in \\ PSYCHOLOGY
}

by

Michael Suarez

2013 
To: Dean Kenneth Furton

College of Arts and Sciences

This thesis, written by Michael Suarez, and entitled Predicting Explorative Behavior by Level of Emotional Reactivity in Bobwhite Quail Neonates (Colinus virginianus), having been approved in respect to style and intellectual content, is referred to you for judgment.

We have read this thesis and recommend that it be approved.

Bennett Schwartz

$\overline{\text { Anibal Gutierrez }}$

Robert Lickliter, Major Professor

Date of Defense: October 30, 2012

The thesis of Michael Suarez is approved.

Dean Kenneth Furton
College of Arts and Sciences

Dean Lakshmi N. Reddi University Graduate School

Florida International University, 2013 


\section{ACKNOWLEDGMENTS}

I am incredibly thankful to my supervisor and mentor, Dr. Robert Lickliter, for his guidance, encouragement, and dedication throughout this process. I am also grateful to the MBRS RISE program for providing the resources and support that have helped me complete my project and enhanced my overall research experience. Lastly, I offer my regards to my family, friends, and lab mates (past and present) for their understanding and support during this time. 


\begin{abstract}
OF THE THESIS
PREDICTING EXPLORATIVE BEHAVIOR BY LEVEL OF EMOTIONAL REACTIVITY IN BOBWHITE QUAIL NEONATES (Colinus virginianus) by
\end{abstract}

Michael Suarez

Florida International University, 2013

Professor Robert Lickliter, Major Professor

Tests of emotional reactivity have been used in a broad range of basic and applied research and have been primarily concerned with how rearing conditions, particularly environmental enrichment, can affect reactivity. However, assessment of how emotional reactivity can be altered during testing procedures and how it affects behaviors such as exploration is relatively uncommon. The present study assessed the explorative responses of Northern bobwhite quail (Colinus virginianus) neonates under conditions of either elevated or attenuated emotional reactivity during a maze task. Measures of emotional reactivity were compared with measures of exploration to determine their relationship with one another. Chicks that were highly emotionally reactive were generally less willing to explore during the maze task than chicks that were less emotionally reactive. Results indicate that levels of emotional reactivity and approach/avoidance motivation play a role in the speed and amount of exploration that is likely to occur in novel environments. 


\section{TABLE OF CONTENTS}

CHAPTER

PAGE

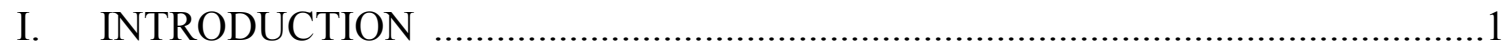

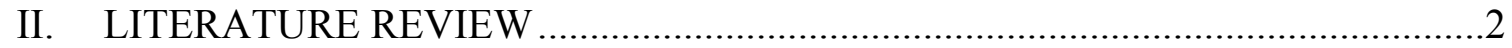

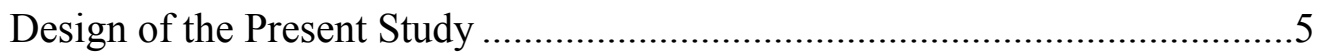

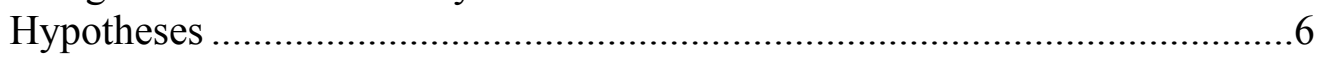

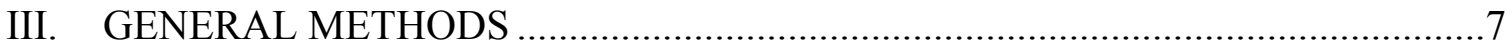

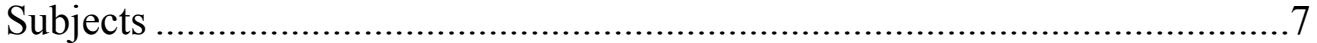

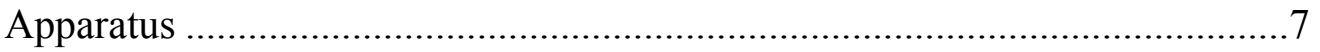

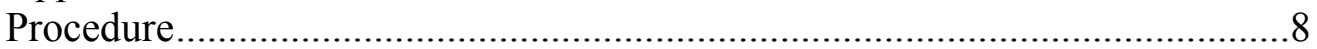

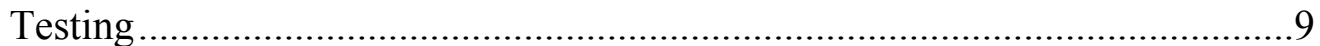

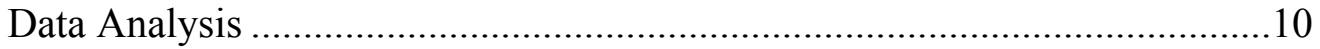

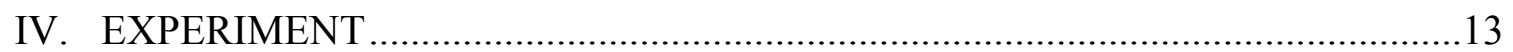

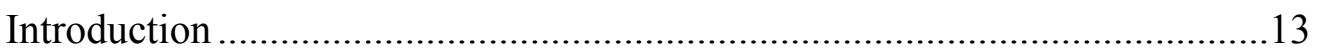

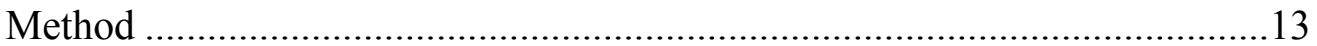

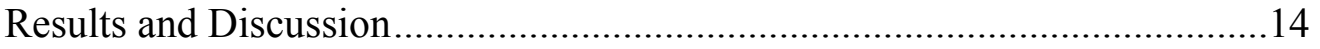

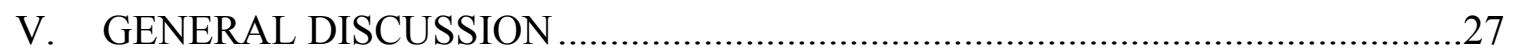

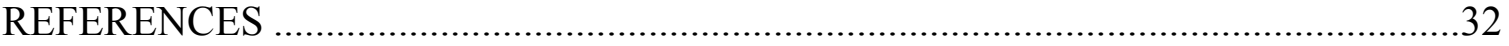




\section{LIST OF TABLES}

TABLE

PAGE

1. Preference Scores for Subjects in the Attractive Test and Aversive Test Conditions .14

2. Duration Scores for Subjects in the Attractive Test and Aversive Test Conditions

3. Mean Rank Duration Scores for Subjects in the Attractive Test and Aversive Test Conditions

4. Measures of Emotional Reactivity:

Immobile Duration

5. Measures of Emotional Reactivity:

Latency to Emerge from Starting Chamber

6. Measures of Emotional Reactivity:

Mean Velocity of Movement

7. Measures of Emotional Reactivity:

Defecation

8. Measures of Exploration:

Percentage of Maze Explored.

9. Measures of Exploration:

Latency to Complete Maze.

10. Measures of Exploration:

Latency to Complete Maze After Emerging from the Starting Chamber.

11. Measures of Exploration:

Total Distance Traveled. 


\section{LIST OF FIGURES}

FIGURE

PAGE

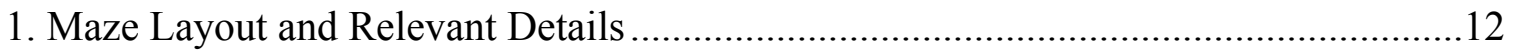

2. Duration Scores for Subjects in the Attractive Test and Aversive Test Conditions

3. Mean Rank Duration Scores for Subjects in the Attractive Test and Aversive Test Conditions... .16

4. Mean Rank Scores for Subjects in the Approach and Avoidance Conditions .

5. Mean Rank Scores for Subjects in the Control 1 and Control 2 Conditions.

6. Mean Rank Scores for Subjects in the Approach and Avoidance Conditions .

7. Mean Rank Scores for Subjects in the

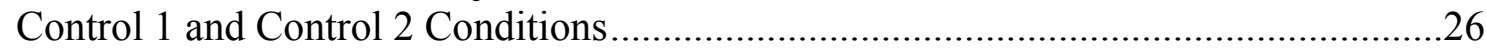




\section{CHAPTER I.}

\section{Introduction}

Research has consistently demonstrated that results observed during testing can be altered in a variety of ways depending on the conditions presented to subjects prior to testing. These alterations are often the result of effects on emotional reactivity, which can be influenced by such things as stressful and isolated living conditions, availability of enriching stimuli, and even the amount of human exposure a subject has during development (Mench, 1992; Hernsworth, Barnett, \& Jones, 1993; Vanderheed \& Bouissou , 1998; Molina-Hernandez, Tellez-Canatara, \& Perez-Garcia, 2001).

Explorative tendencies are also often influenced by the conditions subjects are exposed to prior to testing. Stimulus deprivation prior to testing can lead to decreased spatial exploration behavior and general inactivity (Sackett, 1965), whereas physically enriching conditions often lead to increased spatial exploration, navigational skills, and overall activity during testing (Freire, Cheng, \& Nicol, 2004; Miller \& Mench, 2005; Lazic, Schneider, \& Lickliter, 2007).

Although a great deal of work has been done to explore how rearing conditions affect emotional reactivity and exploration, little is known about determinants that could be present during testing that could have significant effects on reactivity levels and motivation to explore novel surroundings during testing. The present study aimed to fill the gap in the literature by exploring if level of emotional reactivity could be elevated or attenuated during testing, and by observing how this difference in level of emotional reactivity would influence explorative behavior in bobwhite quail (Colinus virginianus) neonates. 


\section{CHAPTER II.}

\section{Literature Review}

The term "emotional reactivity" is often used interchangeably with the term "fearfulness" which has been defined as the general susceptibility of an individual to react to potentially threatening situations (Boissy, 1995). Fearfulness has been proposed to be a personality trait across a variety of animal species, including birds, mammals, amphibians, and invertebrates, and is often assumed to be stable over time (e.g., Goddard \& Beilharz, 1984; Mills \& Faure, 1986, 2000; Jones, 1988; Lyons, 1989; Boissy \& Bouissou, 1995), although some have argued that fearfulness may be more context specific and thereby more variable over time than is often assumed (Miller, Garner, \& Mench, 2006).

Measures of emotional reactivity are often used as indicators of animal welfare, with the premise that highly reactive individuals have been subject to intense and prolonged arousal by means of stressful living conditions (Mench, 1992; Vanderheed \& Bouissou, 1998). For example, it has been shown that isolated rearing conditions can lead to increased signs of emotional reactivity such as fear, depressive-like behaviors, and hyperactivity in rats during open field tasks (Molina-Hernandez et al., 2001). Even minor differences in rearing conditions, such as the type of rearing receptacle that the subject inhabits, the position within the rearing room, and the amount of human exposure during development, can have significant effects on levels of emotional reactivity (Hernsworth et al., 1993). It has also been shown that levels of emotional reactivity could be lessened during testing through the enrichment of rearing conditions prior to testing in a variety of species, including piglets (Bolhuis, Schouten, de Leeuw, Schrama, \& Wiegant, 2004), 
ewes (Vandenheede \& Bouissou, 1998), domestic chickens (Freire et al., 2004; Jones, 2002), crimson-bellied conures (van Hoek \& King, 1997) and Japanese quail (Miller \& Mench, 2005).

The effects of differential rearing conditions on exploration have also been investigated using several different animal models and rearing conditions. Stimulus deprivation during early development was shown to result in rhesus monkeys that lack spatial exploration behavior and are generally inactive when compared to monkeys that are brought up in typical lab conditions (Sackett, 1965). In contrast, physically enriching rearing conditions have shown to produce increased levels of foraging and explorative activities in Japanese quail (Miller \& Mench, 2005) and domestic chickens (Jones, 2002). The addition of hiding places or burrowing tubes to otherwise barren rearing conditions has also shown to significantly increase spatial exploration and navigational skills in subsequent maze tasks (Freire et al., 2004; Lazic et al., 2007).

A widely used method for measuring emotional reactivity in animal subjects is some form of behavioral assessment or testing. Behavioral tests typically focus on recording a subject's behavioral reactions to novel or startling stimuli and often takes place in novel environments that are unfamiliar to the subject being tested (Manteca \& Deag, 1993; Boissy, 1995; Miller, Garner, \& Mench, 2005). In quail species in particular, behavioral tests of emotional reactivity include measures of latency to explore novel areas, latency to explore novel objects, latency to taste novel foods, and reaction to surprise tests (Miller et al., 2005). These tests are particularly useful with quail subjects because they often "freeze" as a fear response, as opposed to other behaviors that may be more difficult to assess. 
Another method for measuring emotional reactivity in animals is through the analysis of products left behind after testing is completed. The most common example of this measurement of emotional reactivity is the amount of feces left behind by the subject after testing (van der Staay et al., 2009). Imada (1970) found that Maudsley Reactive rats defecated significantly more than nonreactive strains of rats during open-field testing for emotionality and concluded that defecation could serve as an index of fear to a certain extent.

Precocial avian species such as bobwhite quail (Colinus virginianus), Japanese quail (Coturnix japonica), and domestic chickens (Gallus gallus) have been identified as animal species that are well suited for assessing the influence of emotional reactivity on an array of behaviors (Jones, 2002; Freire, Cheng, \& Nicol, 2004; Miller et al., 2005). These species are particularly useful in this field of research because they are precocial, which offers a unique experimental opportunity because they are able to be active agents within the testing environment immediately after hatching, unlike rodents or other altricial mammals that are born without fully developed sensory or locomotor systems.

Motivation for exploration can take two different forms, depending on the stimuli present, the state of the organism, and the circumstances or features of the environment; these are approach motivation and avoidance motivation (Schneirla, 1959, 1965; Elliot \& Covington, 2001). Approach motivation refers to the energization of behavior toward positive or desirable stimuli with the intent of getting closer to the stimuli or keeping the stimuli close to the organism. Approach motivation is the most common form of motivation used during maze testing. Avoidance motivation refers to the energization of behavior away from negative or aversive stimuli, with the intent of distancing or keeping 
the stimuli away from the organism (Elliot \& Covington, 2001; Elliot, 2006). Theodore Schneirla $(1959,1965)$ proposed that during early stages of development, there is a direct relationship between the intensity of a stimulus and the direction in which an organism will move with respect to the location of that stimulus. More specifically, he proposed that organisms are more likely to approach low-intensity stimuli and withdraw from highintensity stimuli if they have no prior experience with the stimuli or their potential consequences.

The effects of aversive stimuli on a subject's willingness to explore novel environments has seldom been reviewed in the literature. Aversively motivated tasks were shown to increase emotional reactivity and inhibit performance in a Morris water maze in some rat strains, although it has not been determined if this increase in emotional reactivity has any effect on cognitive performance or if it is generalizable to other animal species or testing conditions (Mowrer, 1939; van der Staay et al. 2009). There is much work that should be done on this topic to extrapolate how aversive stimuli affect subjects during testing.

\section{Design of the Present Study}

The present study uses animal subjects (bobwhite quail) to determine how emotional reactivity can be either elevated or attenuated during testing and to observe how altering reactivity level affects explorative behavior within a novel maze environment. As previously reviewed, the majority of research in the areas of emotional reactivity and exploration has focused on factors that take place throughout development prior to testing and have lasting effects that carry over into testing trials. The current study aims to keep all developmental variables constant across experimental groups to 
focus on determinants within a testing trial that may affect emotional reactivity, and in turn, explorative tendencies. The present study tested bobwhite quail neonates 24 hours after hatching to keep variability caused by extraneous environmental factors that may occur prior to testing to a minimum. With this control in place, this study is more confidently able to conclude that any observed effects are the result of differences present during experimental trials and not a result of variations in conditions prior to testing.

During testing, chicks were individually placed within a novel maze apparatus either in the presence of a continually playing maternal call auditory stimulus or a novel tone auditory stimulus. These stimuli were tested to ensure that they were in fact generally attractive and aversive respectively to the subjects being used. Each trial ran for 1200 seconds during which a randomly allocated chick either explored the maze in the presence of the species-typical bobwhite maternal call, or in the presence of a novel tone, depending on experimental condition. Measures of emotional reactivity and explorative behavior were tracked throughout each trial and subsequently compared to one another.

\section{Hypotheses}

It was hypothesized that the introduction of the maternal call auditory stimulus during experimental trials would serve to decrease level of emotional reactivity while serving to increase explorative behavior in a maze task. In contrast, the introduction of the novel tone auditory stimulus was hypothesized to increase level of emotional reactivity, thereby decreasing explorative behavior in the same maze task. This hypothesis was in line with findings that showed that aversively motivated tasks increased emotional reactivity while inhibiting exploration in some rat strains (van der Staay, et al., 2009). 


\section{CHAPTER III.}

\section{General Methods}

\section{Subjects}

Subjects were 154 incubator reared bobwhite quail chicks (Colinus virginianus)

divided among six conditions. Fertilized, unincubated eggs were received weekly from a commercial game bird supplier (Stickland) and set in a custom-built incubator maintained at $37.5^{\circ} \mathrm{C}$, with a relative humidity of $75-80 \%$. Following hatching, chicks were transferred into standard plastic rearing tubs in groups of 12 to replicate typical brood conditions, and placed in a sound-proof rearing room maintained at approximately $30^{\circ} \mathrm{C}$. Food and water were available ad libitum except during testing. To control for possible between-batch biases, subjects were drawn for each condition across several weeks. Only those subjects that hatched on the 23rd day of incubation were tested in this study, rearing tubs were always placed in approximately the same position across weeks, and the amount of human interaction prior to testing was kept relatively constant.

\section{Apparatus}

Behavioral tests were conducted using a $58.42 \mathrm{~cm}$ by $58.68 \mathrm{~cm}$ square maze set inside a sound attenuated room (see Figure 1). The maze was divided into 5 parallel chambers of equal area, each of which measured $58.42 \mathrm{~cm}$ by $11.74 \mathrm{~cm}$. Each dividing panel had an $8 \mathrm{~cm}$ by $9.5 \mathrm{~cm}$ opening cut out at the end opposite of where the subject could enter each chamber. One wall of the maze was hollowed out and replaced with a wire screen (see Figure 1) to allow sound to pass through the maze more easily. A small speaker concealed behind the wire screen on the outside of the maze was used to present auditory stimuli during each experimental trial. A video camera was mounted directly 
above the maze and connected to a computer located outside of the testing room. Noldus Ethovision XT tracking software was used to automatically record several behavioral measures, including total distance traveled, percentage of maze explored, and latency to enter and exit each chamber during the course of a trial.

Procedure

Bobwhite quail chicks were randomly allocated to one of six experimental conditions following hatching: $(1)$ an Attraction Test condition $(\mathrm{n}=17)$ used to determine whether the bobwhite maternal call being used was generally attractive to the chicks, (2) an Aversive Test condition $(n=17)$ used to determine whether the novel tone stimulus being used was generally aversive to the chicks, (3) an Approach condition (n = 30) in which level of emotional reactivity was decreased through the introduction of the bobwhite maternal call and in which chicks were observed as they explored in the direction of the call being played, (4) an Avoidance condition $(n=30)$ in which level of emotional reactivity was increased through the introduction of the novel tone stimulus and in which chicks were observed as they explored in the direction opposite of the tone being played, and two control conditions $(n=30 /$ per condition) that were used to ensure there were no starting side biases within the maze.

All subjects were tested individually 24 hours after hatch, starting at approximately $12 \mathrm{pm}$ each week to control for developmental age and other potential daily rhythm variables. To avoid any effects that may arise from social isolation prior to testing, the last four birds in each rearing tub were not tested. Each subject was transferred from the rearing room by hand and placed at its corresponding starting point, at which time the auditory stimulus immediately began playing and Noldus Ethovision 
XT automatically began recording all subsequent movement throughout the session. Each trial ran for 1200 seconds $(20 \mathrm{~min})$ to give ample time for subjects in each condition to explore the maze, without limiting those that were more emotionally reactive and thus slower to respond (Gray, 1990). After testing, each subject was transferred back to the rearing room and placed in a new rearing tub isolated from those subjects that had yet to be tested. The number and relative size of defecation was noted for each subject following the trial and then removed completely from the testing surface.

Testing

Each of the six testing conditions outlined previously had unique testing components while maintaining identical pre-test conditions. In the Attraction Test condition (1), a chick was placed at the end of the maze closest to the speaker at the start of each trial (see Figure 1) as a species-typical bobwhite maternal call recording (Heaton, Miller, \& Goodwin, 1978), calibrated to $65 \mathrm{~dB}$ at the subject's starting point, was played continually from the speaker located behind the wire mesh for the entire duration of the trial. In the Aversive Test condition (2), a chick was placed at the end of the maze furthest from the speaker at the start of each trial (see Figure 1) as a $120 \mathrm{~Hz}$ novel tone auditory stimulus, calibrated to $65 \mathrm{~dB}$ at the subject's starting point, was played continually at a rate of 15 tones per minute from the speaker located behind the wire mesh for the entire duration of the trial. The purpose of these preliminary tests was to determine that the auditory stimuli being used were generally attractive and aversive, respectively, to the chicks under these testing conditions.

In the Approach condition (3), a chick was placed at the end of the maze furthest from the speaker at the start of each trial (see Figure 1) as the same maternal call used in 
the Attraction Test condition was played continually from the speaker located behind the wire mesh for the entire duration of the trial. In the Avoidance condition (4), a chick was placed at the end of the maze closest to the speaker at the start of each trial (see Figure 1) as the same novel tone used in the Aversive Test condition was played continually at a rate of 15 tones per minute from the speaker located behind the wire mesh for the entire duration of the trial. The purpose of these experimental conditions was to demonstrate how level of emotional reactivity and explorative behavior could be altered within testing trials.

Two control conditions were used to ensure that there were no starting biases within the maze that could affect the results of the previous conditions. The first control condition (5) shared identical testing conditions to both the Aversive Test and Approach conditions with the exception that no auditory stimuli were played from the speaker at any time throughout the testing trial. The second control condition (6) shared identical testing conditions to both the Attractive Test and Avoidance conditions with the exception that no auditory stimuli were played from the speaker at any time throughout the testing trial.

Data Analysis

The relevant dependent variable for the Attractive Test (1) and Aversive Test (2) conditions was preference to stay either in close proximity or extended proximity from the auditory stimulus present during each testing trial. The measures of preference used were 1) "close proximity"- total duration of time spent in the two chambers closest to the speaker playing the auditory stimulus and 2) "extended proximity"- total duration of time spent in the two chambers furthest from the speaker playing the auditory stimulus. A 
preference was determined to exist if a chick spent at least $50 \%$ more time in close vs. extended proximity (or vice-versa) to the auditory stimulus present. Time spent in the third chamber of the maze was considered neutral space and did not count toward displaying preference for either side.

The relevant dependent variables for the Approach, Avoidance, and both control conditions were the measures of emotional reactivity and exploration during the testing trials. The measures of emotional reactivity were 1) time spent immobile, 2) latency to exit the starting chamber of the maze into a novel chamber, 3) mean velocity, and 4) total instances of defecation throughout the trial. The measures of exploration were 1) percentage of maze explored, 2) time to maze completion, 3) time to maze completion after emerging into the 2 nd chamber of the maze, and 4) total distance traveled throughout the trial.

Duration scores within the Attractive Test and Aversive Test conditions were evaluated using the Wilcoxin Signed Ranks test. Individual preferences were evaluated using the Chi-Square test. Significance levels of $\mathrm{p} \leq .05$ (two-tailed) were used to evaluate these results. Each measure of emotional reactivity and exploration across the Approach, Avoidance, and both control conditions were tested for normality using the Shapiro-Wilk test and subsequently evaluated using the non-parametric Mann-Whitney U test. Due to the preliminary results found in the Attractive Test and Aversive Test conditions as well as the directional hypotheses made in this study, significance levels of $\mathrm{p} \leq .05$ (one-tailed) were used to evaluate the results of the Approach and Avoidance conditions. Significance levels of $p \leq .05$ (two-tailed) were used to evaluate the results of both control conditions. 
Figure 1: Maze Layout and Relevant Details

C.

A.

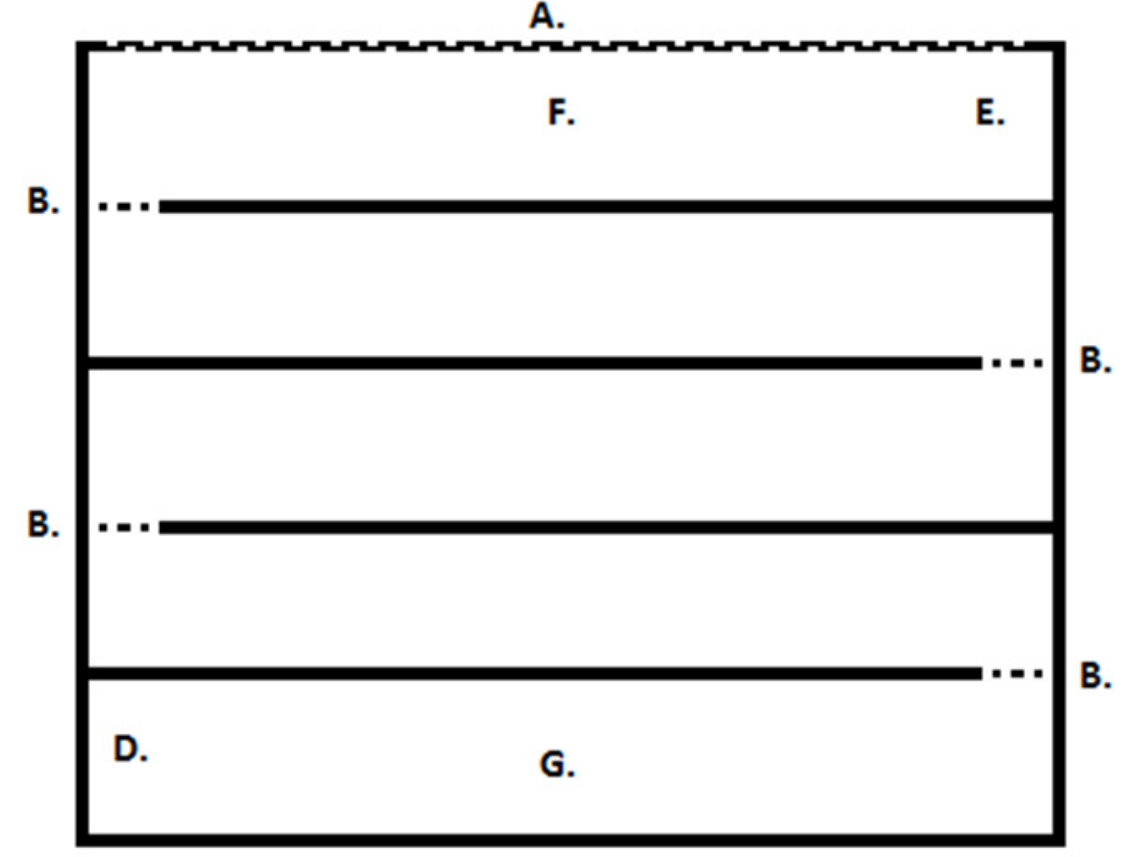

Legend: $\quad$ A. Wire Screen

B. $8 \mathrm{~cm}$ by $9.5 \mathrm{~cm}$ Openings

C. Speaker Location

D. Aversive Test, Approach, \& Control 1 Conditions Starting Locations

E. Attraction Test, Avoidance, \& Control 2 Conditions Starting Locations

F. Approach \& Control 1 End Point (90\% Maze Completion)

G. Avoidance \& Control 2 End Point (90\% Maze Completion) 
CHAPTER IV.

\section{Experiment}

\section{Introduction}

To investigate how level of emotional reactivity and explorative behavior could be modified within a testing trial, bobwhite quail neonates were tested at 24 hours of age in a maze task while a series of behavioral measures were observed. I hypothesized that level of emotional reactivity could be either attenuated or elevated through the introduction of auditory stimuli during testing trials. I also hypothesized that chicks whose level of emotional reactivity was attenuated would be more willing to explore their novel maze surroundings, whereas chicks whose level of emotional reactivity was elevated would be less willing to explore the same novel maze surroundings during testing.

Method

One hundred and fifty-four bobwhite quail neonates, divided into 6 conditions (Attractive Test $\mathrm{n}=17$; Aversive Test $\mathrm{n}=17$; Approach $\mathrm{n}=30 ;$ Avoidance $\mathrm{n}=30$; Control-1 $n=30$; Control-2 $n=30$ ), served as subjects. The Attractive Test condition served to demonstrate how the bobwhite maternal call used in this study works to generally attract chicks toward its direction. The Aversive Test condition served to show how the novel tone used in this study works as an aversive stimulus that chicks generally tend to prefer to stay away from. The Approach condition presented the maternal call stimulus to reduce emotional reactivity levels and motivate exploration in the direction of the call. The Avoidance condition presented the aversive novel tone stimulus to elevate emotional reactivity levels and to motivate exploration in the opposite direction of the 
tone. Both control conditions served to demonstrate how chicks would act within the maze in the absence of any auditory stimuli and to ensure that there were no inherent starting side biases within the maze. All groups were tested at 24 hours after hatching (see General Methods for details).

Results and Discussion

Results for the Attractive Test and Aversive Test conditions are shown in Tables 1,2 , and 3 . These results indicate that chicks generally have a preference for staying in close proximity to the bobwhite maternal call $\left(\chi^{2}=13.24, p<.001\right.$, two-tailed), whereas chicks generally have a preference for staying at an extended proximity to the novel auditory tone stimulus $\left(\chi^{2}=10.70, p<.01\right.$, two-tailed). These results also indicate that chicks in the Attractive Test condition spent a significantly greater mean duration in close proximity of the maternal call than at an extended proximity $(\mathrm{Z}=-3.65, p<.001$, twotailed), whereas chicks in the Aversive Test condition spent a significantly greater mean duration at extended proximity of the novel tone auditory stimulus $(Z=-2.68, p<.01$, two-tailed). These mean differences are illustrated in Figures 2 and 3.

Table 1: Preference Scores for Subjects in the Attractive Test and Aversive Test Conditions

\section{Condition n Close Proximity Extended Proximity No Preference}

\begin{tabular}{lcccc}
\hline $\begin{array}{c}\text { Attractive } \\
\text { Test }\end{array}$ & 17 & $16^{*}$ & 0 & 1 \\
$\begin{array}{c}\text { Aversive } \\
\text { Test }\end{array}$ & 17 & 3 & $12^{*}$ & 2 \\
\hline
\end{tabular}

*Significant Preference, $p \leq .05$ (Chi-Square Test) 
Table 2: Duration Scores for Subjects in the Attractive Test and Aversive Test Conditions

\begin{tabular}{cccc} 
Condition & n & Close Proximity & Extended Proximity \\
\hline Attractive Test & 17 & $1078.30 \mathrm{~s}$ & $81.22 \mathrm{~s}$ \\
& & $(191.00)$ & $(146.23)$ \\
Aversive Test & 17 & $308.61 \mathrm{~s}$ & $797.86 \mathrm{~s}$ \\
& & $(300.06)$ & $(332.38)$ \\
\hline
\end{tabular}

Mean scores are shown. Standard deviations are shown in parentheses.

Table 3: Mean Rank Duration Scores for Subjects in the Attractive Test and Aversive Test Conditions

\begin{tabular}{cccc} 
Condition & $\mathbf{n}$ & Close Proximity & Extended Proximity \\
\hline Attractive Test & 17 & $26.00^{*}$ & 9.00 \\
& & $(4.87)$ & $(4.32)$ \\
Aversive Test & 17 & 11.18 & $23.82^{*}$ \\
& & $(7.71)$ & $(7.72)$
\end{tabular}

Mean rank scores are shown. Standard deviations are shown in parentheses.

*Significantly Higher Mean Rank Duration, $p \leq .05$ (Wilcoxin Signed Ranks Test) 
Figure 2: Duration Scores for Subjects in the Attractive Test and Aversive Test Conditions

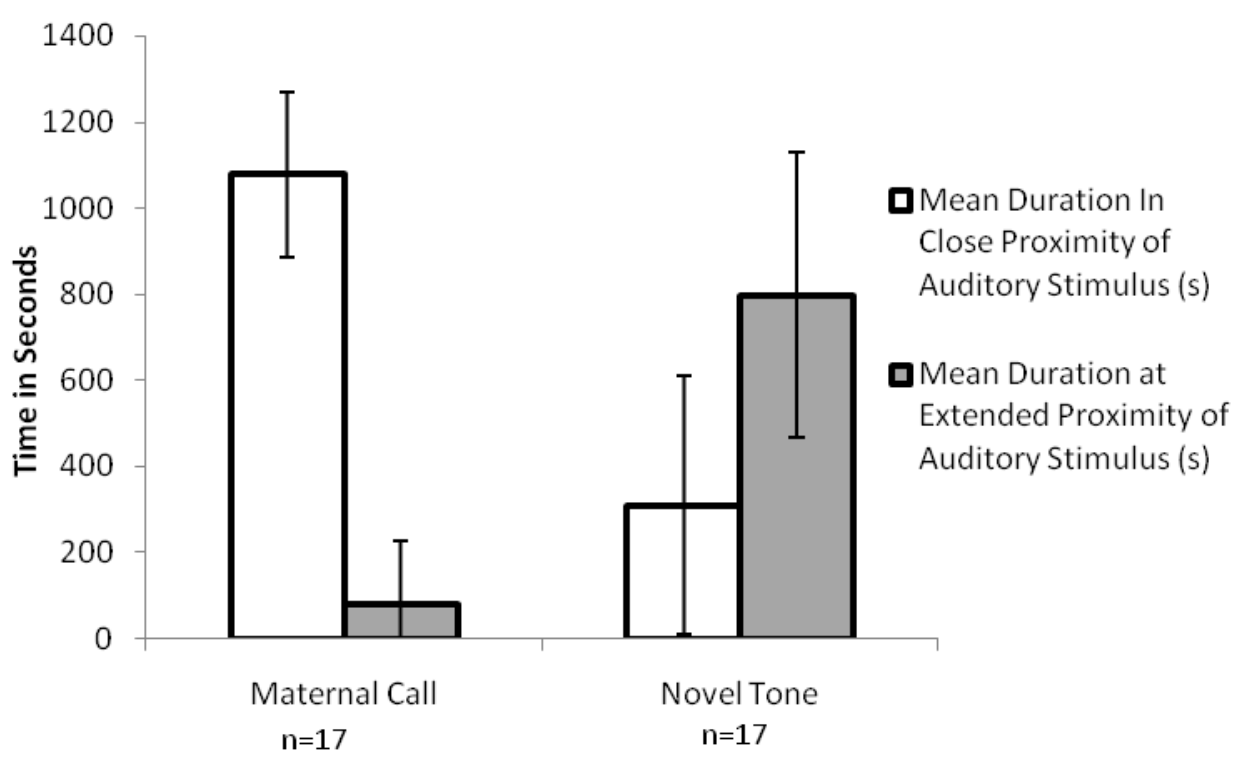

Figure 3: Mean Rank Duration Scores for Subjects in the Attractive Test and Aversive Test Conditions

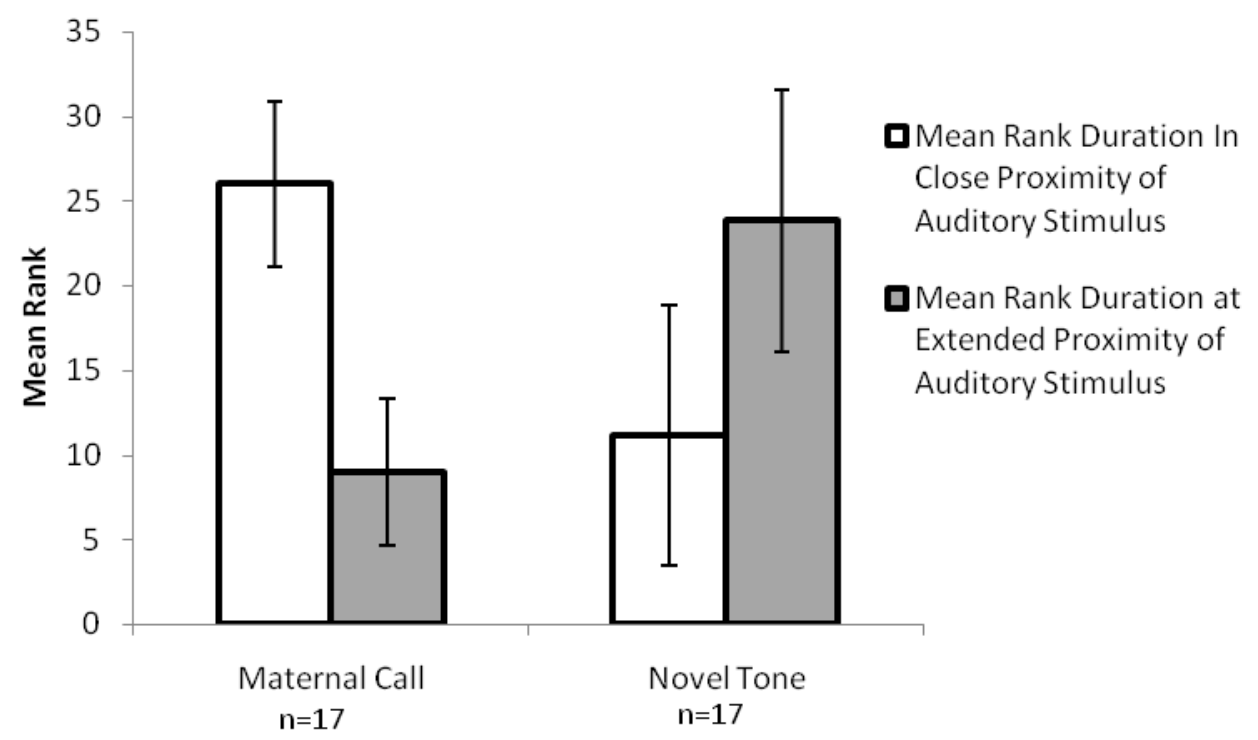


Tables 4 - 7 and Figures 4 - 5 illustrate the results of the measures of emotional reactivity in the Approach, Avoidance, and both control conditions.

The results of the measure of immobile duration are shown in Table 4. These results indicate that chicks in the Approach condition had a significantly shorter immobile duration than the chicks in the Avoidance condition, $\mathrm{U}(58)=331.00, \mathrm{Z}=-1.76$, $p \leq .05$ (one-tailed). The two control groups did not differ significantly, $\mathrm{U}(58)=439.00$, $\mathrm{Z}=-.163, p=.87$ (two-tailed). These mean rank differences are illustrated in Figure 4 and Figure 5.

Table 4: Measures of Emotional Reactivity: Immobile Duration

\begin{tabular}{cccc} 
Condition & $\mathbf{n}$ & Group Mean & Mean Rank \\
\hline Approach & 30 & $792.17 \mathrm{~s}$ & $26.53^{*}$ \\
& & $(216.39)$ & $(16.81)$ \\
Avoidance & 30 & $893.12 \mathrm{~s}$ & $34.47^{*}$ \\
& & $(235.66)$ & $(17.47)$ \\
Control 1 & \multirow{2}{*}{30} & $(219.34)$ & 30.13 \\
& & $814.42 \mathrm{~s}$ & $(17.58)$ \\
Control 2 & \multirow{2}{*}{30} & $(215.15)$ & 30.87 \\
& & & $(17.46)$ \\
\hline
\end{tabular}

Mean rank scores grouped separately (Approach \& Avoidance vs. Control $1 \&$ Control 2) Standard deviations are shown in parentheses

$* \mathrm{p} \leq .05$ (Mann - Whitney U, one-tailed)

The results of the measure of latency to exit the starting chamber of the maze into a novel chamber are shown in Table 5. These results indicate that chicks in the Approach condition had a significantly shorter latency to emerge from the starting chamber of the 
maze than the chicks in the Avoidance condition, $\mathrm{U}(58)=330.50, \mathrm{Z}=-1.77, p \leq .05$ (one-tailed). The chicks in the Control 1 condition had a significantly longer latency to emerge from the starting chamber of the maze than the chicks in the Control 2 condition, $\mathrm{U}(58)=308.50, \mathrm{Z}=-2.09, p \leq .05$ (two-tailed). These mean rank differences are illustrated in Figure 4 and Figure 5.

Table 5: Measures of Emotional Reactivity: Latency to Emerge from Starting Chamber

\begin{tabular}{cccc} 
Condition & $\mathbf{n}$ & Group Mean & Mean Rank \\
\hline Approach & 30 & $263.83 \mathrm{~s}$ & $26.52^{*}$ \\
& & $(385.71)$ & $(16.51)$ \\
Avoidance & 30 & $400.90 \mathrm{~s}$ & $34.48^{*}$ \\
& & $(417.07)$ & $(17.69)$ \\
Control 1 & 30 & $334.59 \mathrm{~s}$ & $35.22^{* *}$ \\
& & $(345.01)$ & $(14.44)$ \\
Control 2 & 30 & $277.08 \mathrm{~s}$ & $25.78^{* *}$ \\
& & $(354.51)$ & $(19.12)$ \\
\hline
\end{tabular}

Mean rank scores grouped separately (Approach \& Avoidance vs. Control $1 \&$ Control 2) Standard deviations are shown in parentheses

$* \mathrm{p} \leq .05$ (Mann - Whitney U, one-tailed)

$* * \mathrm{p} \leq .05$ (Mann - Whitney U, two-tailed)

The results of the measure of mean velocity of movement are shown in Table 6 .

These results indicate that chicks in the Approach condition had a significantly faster mean velocity of movement than the chicks in the Avoidance condition, $\mathrm{U}(58)=326.00$, $\mathrm{Z}=-1.83, p \leq .05$ (one-tailed). The two control groups did not differ significantly, $\mathrm{U}(58)$ $=432.00, \mathrm{Z}=-.266, p=.79$ (two-tailed). These mean rank differences are illustrated in Figure 4 and Figure 5. 


\section{Table 6: Measures of Emotional Reactivity: Mean Velocity of Movement}

\begin{tabular}{cccc} 
Condition & $\mathbf{n}$ & Group Mean & Mean Rank \\
\hline Approach & 30 & $6.75 \mathrm{~cm} / \mathrm{s}$ & $34.63^{*}$ \\
& & $(3.61)$ & $(17.00)$ \\
Avoidance & 30 & $4.79 \mathrm{~cm} / \mathrm{s}$ & $26.37^{*}$ \\
& & $(3.95)$ & $(17.21)$ \\
Control 1 & 30 & $6.73 \mathrm{~cm} / \mathrm{s}$ & 31.10 \\
& & $(3.71)$ & $(17.88)$ \\
Control 2 & 30 & $6.42 \mathrm{~cm} / \mathrm{s}$ & 29.90 \\
& & $(3.57)$ & $(17.32)$
\end{tabular}

Mean rank scores grouped separately (Approach \& Avoidance vs. Control $1 \&$ Control 2) Standard deviations are shown in parentheses

$* \mathrm{p} \leq .05$ (Mann - Whitney U, one-tailed)

The results of the measure of total instance of defecation are shown in Table 7.

These results indicate that chicks in the Approach and Avoidance conditions did not differ significantly in amount of defecation, $\mathrm{U}(58)=415.00, \mathrm{Z}=-.57, p=.26$ (onetailed). The two control groups also did not differ significantly, $U(58)=434.00, Z=-$ $.254, p=.80$ (two-tailed). These mean rank differences are illustrated in Figure 4 and Figure 5. 
Table 7: Measures of Emotional Reactivity:

Defecation

\begin{tabular}{cccc} 
Condition & $\mathbf{n}$ & Group Mean & Mean Rank \\
\hline Approach & 30 & 0.73 & 31.67 \\
& & $(0.83)$ & $(16.29)$ \\
Avoidance & 30 & 0.60 & 29.33 \\
& & $(0.72)$ & $(15.71)$ \\
Control 1 & 30 & 1.13 & 31.03 \\
& & $(.82)$ & $(16.86)$ \\
Control 2 & 30 & 1.07 & 29.97 \\
& & $(.74)$ & $(15.95)$ \\
& & \\
\hline $\begin{array}{l}\text { Mean rank scores grouped separately (Approach \& Avoidance vs. Control 1 \& Control 2) } \\
\text { Standard deviations are shown in parentheses } \\
* \mathrm{p} \leq .05 \text { (Mann - Whitney U, one-tailed) }\end{array}$
\end{tabular}

Figure 4: Mean Rank Scores for Subjects in the Approach and Avoidance Conditions

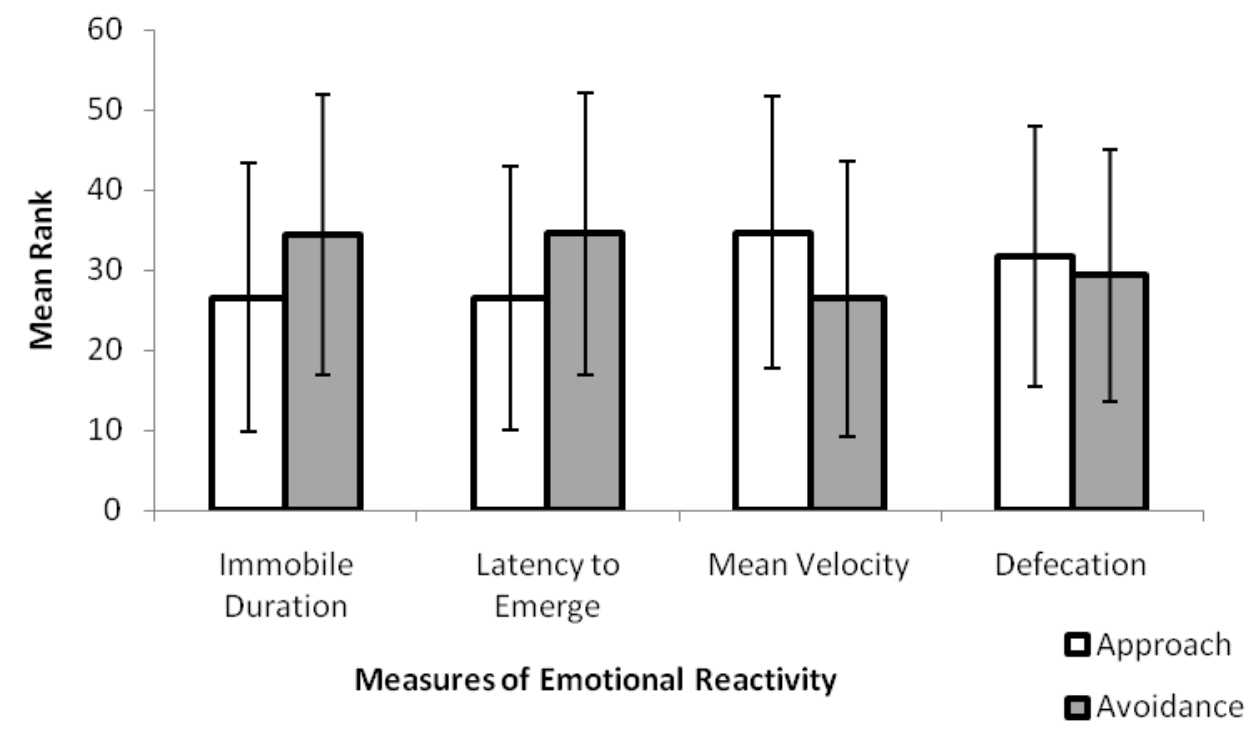


Figure 5: Mean Rank Scores for Subjects in the Control 1 and Control 2 Conditions

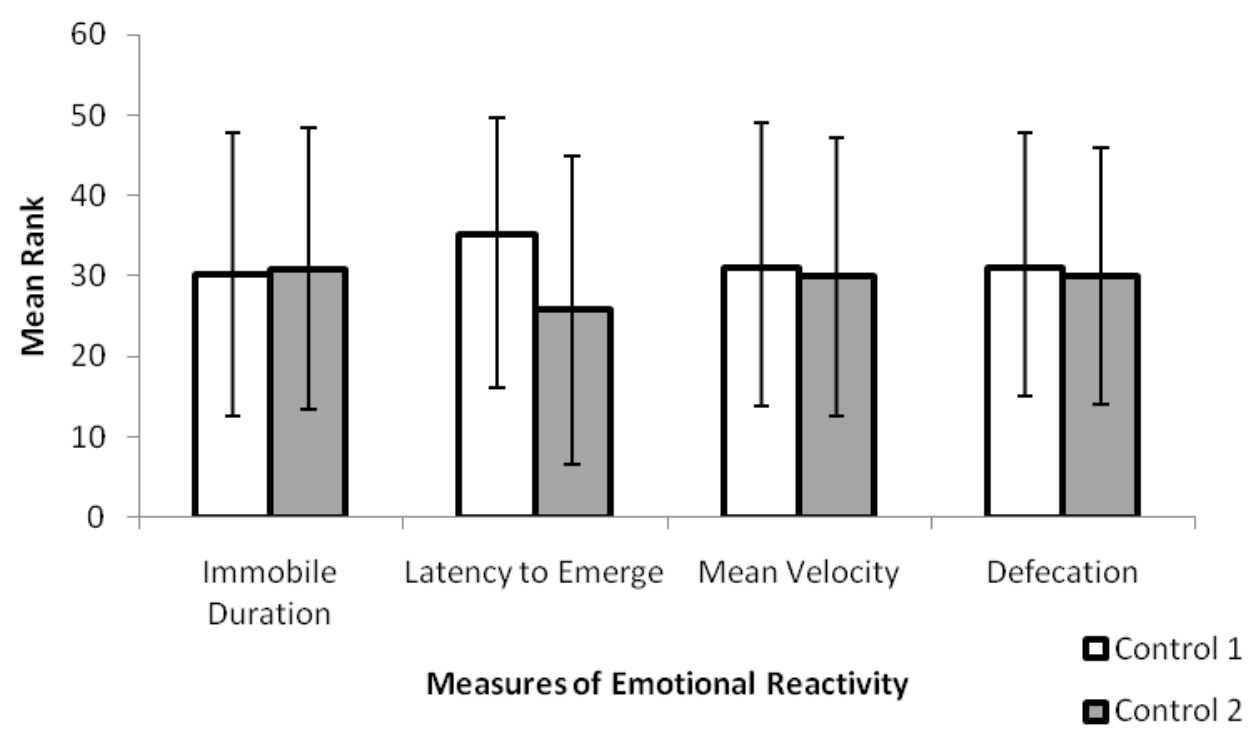

The results of the measures of exploration in the Approach, Avoidance, and both control conditions are shown in Tables 8 - 11 and illustrated in Figures 6 and 7.

The results of the measure of percentage of maze explored are shown in Table 8. These results indicate that chicks in the Approach condition explored a significantly greater proportion of the maze than the chicks in the Avoidance condition, $\mathrm{U}(58)=$ 339.50, $\mathrm{Z}=-1.63, p \leq .05$ (one-tailed). The two control groups did not differ significantly, $\mathrm{U}(58)=413.50, \mathrm{Z}=-.54, p=.59$ (two-tailed). These mean rank differences are illustrated in Figure 6 and Figure 7. 
Table 8: Measures of Exploration:

Percentage of Maze Explored

\begin{tabular}{cccc} 
Condition & $\mathbf{n}$ & Group Mean & Mean Rank \\
\hline Approach & 30 & $85.55 \%$ & $34.18^{*}$ \\
& & $(32.85)$ & $(15.98)$ \\
Avoidance & 30 & $70.04 \%$ & $26.82 *$ \\
& & $(37.70)$ & $(18.36)$ \\
Control 1 & 30 & $85.98 \%$ & 29.28 \\
& & $(27.95)$ & $(18.18)$ \\
Control 2 & 30 & $85.69 \%$ & 31.72 \\
& & $(28.67)$ & $(16.94)$
\end{tabular}

Mean rank scores grouped separately (Approach \& Avoidance vs. Control $1 \&$ Control 2) Standard deviations are shown in parentheses

$* \mathrm{p} \leq .05$ (Mann - Whitney U, one-tailed)

The results of the measure of latency to complete maze are shown in Table 9.

These results indicate that chicks in the Approach condition completed the maze in significantly less time than the chicks in the Avoidance condition, $U(58)=274.50, \mathrm{Z}=-$ 2.63, $p \leq .01$ (one-tailed). The two control groups did not differ significantly, $\mathrm{U}(58)=$ 432.00, $\mathrm{Z}=-.27, p=.79$ (two-tailed). These mean rank differences are illustrated in Figure 6 and Figure 7. 
Table 9: Measures of Exploration:

Latency to Complete Maze

\begin{tabular}{cccc} 
Condition & $\mathbf{n}$ & Group Mean & Mean Rank \\
\hline Approach & 30 & $463.17 \mathrm{~s}$ & $24.65^{*}$ \\
& & $(412.21)$ & $(15.75)$ \\
Avoidance & 30 & $794.47 \mathrm{~s}$ & $36.35^{*}$ \\
& & $(450.79)$ & $(16.88)$ \\
Control 1 & 30 & $686.06 \mathrm{~s}$ & 29.90 \\
& & $(407.36)$ & $(18.03)$ \\
Control 2 & 30 & $697.43 \mathrm{~s}$ & 31.10 \\
& & $(390.70)$ & $(16.82)$
\end{tabular}

Mean rank scores grouped separately (Approach \& Avoidance vs. Control $1 \&$ Control 2) Standard deviations are shown in parentheses

$* \mathrm{p} \leq .05$ (Mann - Whitney U, one-tailed)

The results of the measure of latency to complete maze after emerging from the starting chamber of the maze are shown in Table 10. These results indicate that chicks in the Approach condition completed the maze in significantly less time after emerging into the second chamber of the maze than the chicks in the Avoidance condition, $U(58)=$ 331.00, $Z=-1.76, p \leq .05$ (one-tailed). The two control groups did not differ significantly, $\mathrm{U}(58)=404.50, \mathrm{Z}=-.67, p=.50$ (two-tailed). These mean rank differences are illustrated in Figure 6 and Figure 7. 
Table 10: Measures of Exploration:

Latency to Complete Maze After Emerging from the Starting Chamber

\begin{tabular}{cccc} 
Condition & $\mathbf{n}$ & Group Mean & Mean Rank \\
\hline Approach & 30 & $362.01 \mathrm{~s}$ & $26.53^{*}$ \\
& & $(434.53)$ & $(17.00)$ \\
Avoidance & 30 & $593.74 \mathrm{~s}$ & $34.47^{*}$ \\
& & $(457.18)$ & $(17.23)$ \\
Control 1 & 30 & $473.60 \mathrm{~s}$ & 28.98 \\
& & $(411.73)$ & $(17.49)$ \\
Control 2 & 30 & $540.35 \mathrm{~s}$ & 32.02 \\
& & $(434.31)$ & $(17.59)$
\end{tabular}

Mean rank scores grouped separately (Approach \& Avoidance vs. Control $1 \&$ Control 2) Standard deviations are shown in parentheses

$* \mathrm{p} \leq .05$ (Mann - Whitney U, one-tailed)

The results of the measure of total distance traveled are shown in Table 11. These results indicate that chicks in the Approach condition traveled a significantly longer distance than the chicks in the Avoidance condition, $\mathrm{U}(58)=321.00, \mathrm{Z}=-1.91, p \leq .05$ (one-tailed). The two control groups did not differ significantly, $U(58)=432.00, \mathrm{Z}=$ $.266, p=.79$ (two-tailed). These mean rank differences are illustrated in Figure 6 and Figure 7. 
Table 11: Measures of Exploration:

Total Distance Traveled

\begin{tabular}{cccc} 
Condition & $\mathbf{n}$ & Group Mean & Mean Rank \\
\hline Approach & 30 & $8105.50 \mathrm{~cm}$ & $34.80^{*}$ \\
& & $(4324.84)$ & $(16.96)$ \\
Avoidance & 30 & $5616.92 \mathrm{~cm}$ & $26.20^{*}$ \\
& & $(4693.34)$ & $(17.17)$ \\
Control 1 & 30 & $8071.27 \mathrm{~cm}$ & 31.10 \\
& & $(4460.56)$ & $(17.88)$ \\
Control 2 & 30 & $7696.71 \mathrm{~cm}$ & 29.90 \\
& & $(4276.52)$ & $(17.32)$ \\
& &
\end{tabular}

Figure 6: Mean Rank Scores for Subjects in the Approach and Avoidance Conditions

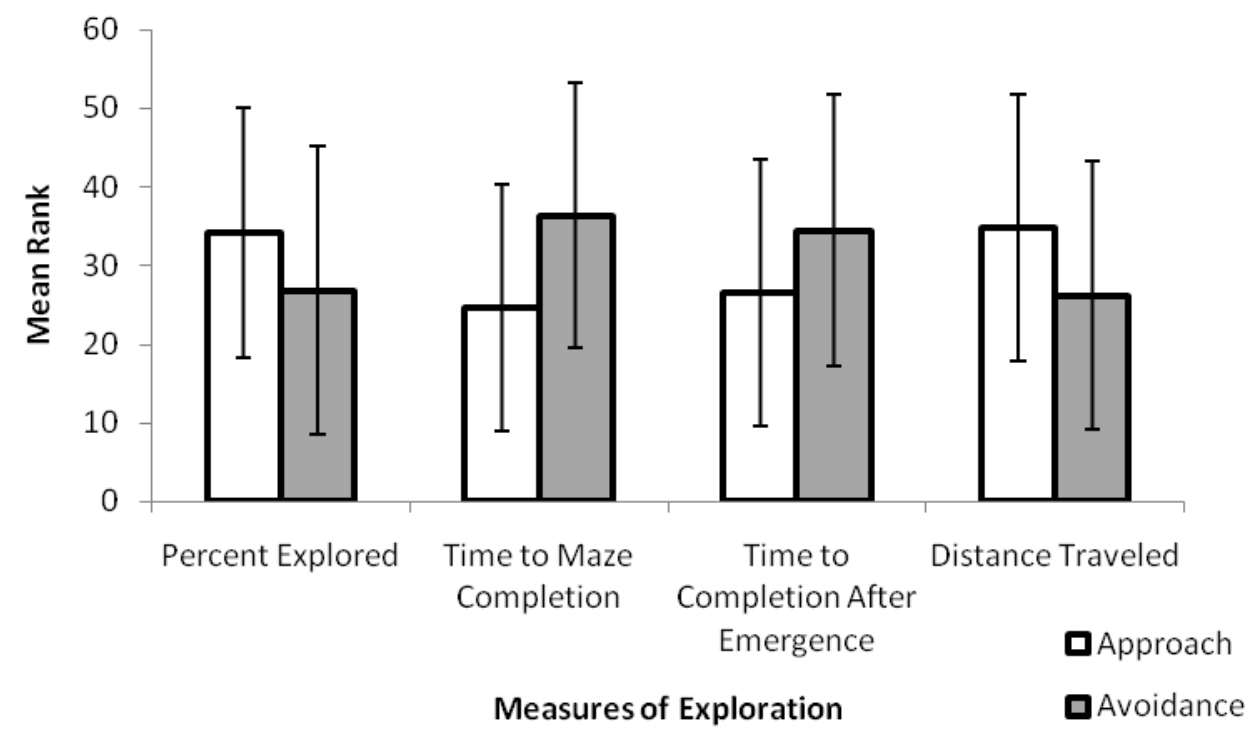


Figure 7: Mean Rank Scores for Subjects in the Control 1 and Control 2 Conditions

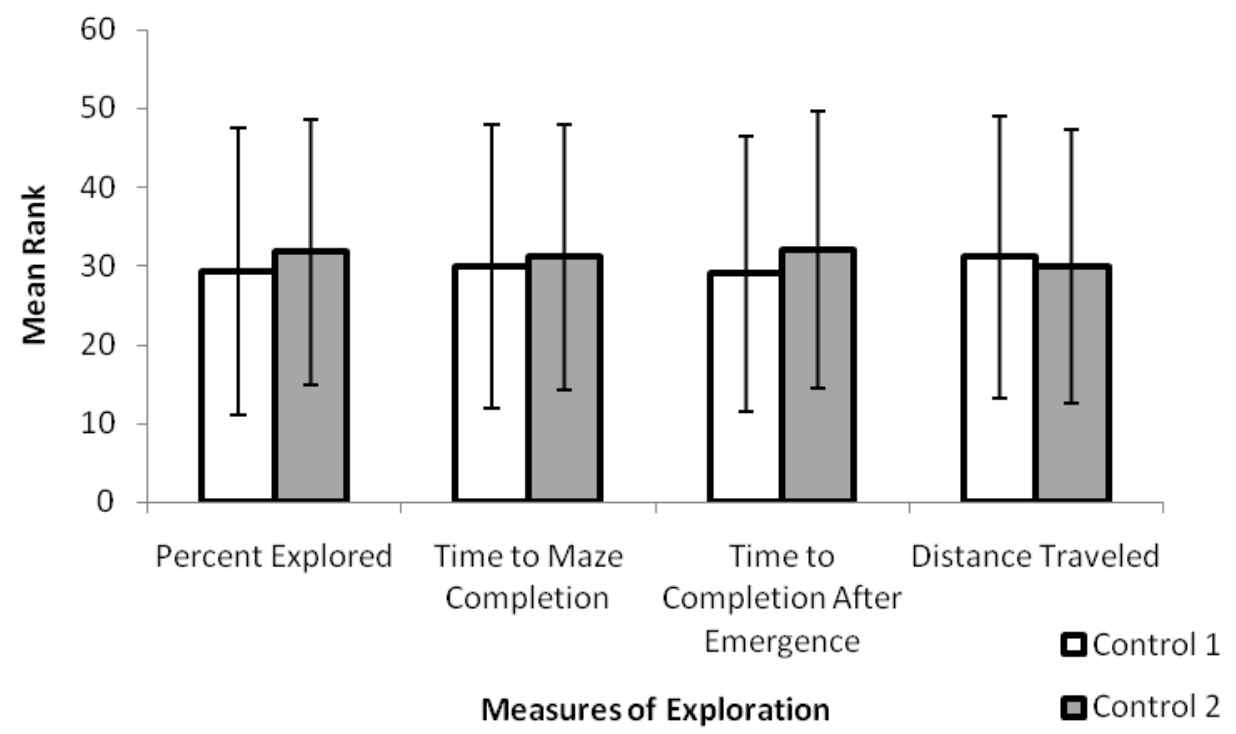

CHAPTER $V$. 


\section{General Discussion}

The main goals of the present study were to identify behavioral indicators of emotional reactivity in Northern bobwhite quail (Colinus virginianus) neonates, explore whether exposure to attractive and aversive auditory stimuli present during testing could alter the level of these behavioral indicators of emotional reactivity, and to assess how reactivity level influences motivation to explore a novel maze environment under these varying conditions. The present results were able to identify three viable behavioral indicators of emotional reactivity (immobile duration, latency to emerge from the starting chamber of the maze, and mean velocity of movement) in bobwhite quail neonates that can be used under similar testing conditions, and also identified amount of defecation as not being a viable measure for this subject pool. These results also demonstrated that level of emotional reactivity could be elevated in the presence of an aversive auditory stimulus and attenuated in the presence of an attractive auditory stimulus. Lastly, these results demonstrated that chicks that are highly emotionally reactive are less willing to explore a novel maze environment (based on the percentage of the maze explored, total distance traveled, and two measures of latency to complete the maze) than chicks that are less emotionally reactive.

The initial set of conditions (Attraction Test and Aversive Test) laid the groundwork for subsequent conditions by evaluating the utility of the auditory stimuli being used. These conditions demonstrated that chicks generally had a preference for staying in close proximity of the bobwhite maternal call auditory stimulus, whereas chicks generally had a preference for staying at an extended proximity of the novel tone 
auditory stimulus. These results are a clear indication of the attractive and aversive natures of the auditory stimuli being utilized throughout this study.

In line with typical methods for measuring emotional reactivity in animal subjects by recording behavioral reactions to novel or startling stimuli within novel environments (Manteca \& Deag, 1993; Boissy, 1995; Miller et al., 2005), the chicks in this study displayed significantly elevated levels of emotional reactivity in the presence of the aversive novel tone than did chicks in the presence of the bobwhite maternal call. The measures that demonstrated utility for this task were the total duration of time spent immobile throughout the testing trial, latency to emerge from the starting chamber of the maze into the second chamber, and mean velocity of movement throughout the testing trial. Chicks tested in the presence of the aversive novel tone spent a significantly greater amount of time immobile, had a significantly longer latency to emerge from the starting chamber of the maze, and had an all around slower mean velocity of movement than chicks that were tested in the presence of the bobwhite maternal call. The increased level of emotional reactivity demonstrated by these measures support the research of van der Staay and colleagues (2009), in which they found that level of emotional reactivity was increased in some rat strains during aversively motivated tasks.

It is important to note that the Control 1 and Control 2 conditions revealed a starting bias within the maze in favor of the Avoidance condition (Table 5). These results demonstrated that in the absence of any auditory stimulation, chicks in the Control 2 condition (same starting location as those in the Avoidance and Attraction Test conditions) took significantly less time to emerge from the starting chamber of the maze than did those in the Control 1 condition. This starting bias, although not optimal, should 
not take away from the present results as the opposite effects were found with the introduction of the auditory stimuli. That is, chicks in the Approach condition took significantly less time to emerge from the starting chamber of the maze than did those in the Avoidance condition, even though the control conditions showed a bias in the opposite direction.

Amount of defecation was also explored as a possible indicator of emotional reactivity, but it did not differ significantly between chicks that were tested in the presence of the bobwhite maternal call or the aversive novel tone. There are several reasons for why this measure may be useful for other animal species, but not for the chicks used in this study. First, the chicks used in this study were tested twenty-four hours after hatch and they were still being sustained to a large extent by nutrition absorbed from the egg yolk and stored prenatally. Another possible explanation for why defecation may not have differed between these two groups has to do with the "freeze" response that these chicks demonstrate, to varying degrees, immediately after being placed within the maze at the start of each trial. As shown in Table 5, the chicks in the Approach condition spent less time "frozen" than the chicks in the Avoidance condition. I observed that during this "frozen" duration, chicks were not at all likely to defecate and that all of the defecation that was recorded in this study occurred after this "freeze" duration had ended. This anecdotal finding leads me to believe that there may be a difference in the utility of the defecation measure depending on whether an animal subject is a "freezer" (an animal that is more likely to remain undetected in the presence of a predator by remaining as still as possible) or a "fleer" (an animal that is more likely 
to escape a predator by fleeing), although there is no research available with regard to this measure to corroborate this hypothesis.

The present study assessed how level of emotional reactivity affected motivation to explore a novel maze environment through the use of four explorative measures. These were percentage of maze explored, latency to complete the maze, latency to complete the maze after emerging from the starting chamber, and total distance traveled throughout the testing trial. My study demonstrated that chicks were more motivated to explore the maze across all four of these measures while in the presence of the bobwhite maternal call than in the presence of the aversive novel tone indicating that as level of emotional reactivity increases, willingness to explore decreases. This assertion may again only apply to "freezers" and not necessarily to "fleers", who may be more motivated to escape from an aversive stimulus than to try to remain undetectable in its presence.

It should be noted that latency to complete the maze was measured in two different ways. The first method for measuring latency to complete the maze included all of the time from the start of the trial, to the point at which the chick reached the end of the maze, which in this case was determined to be the $90 \%$ maze completion mark. A second method for measuring latency to complete the maze was implemented to account for the time spent in a "highly aroused" state, during which chicks were either "frozen" or unwilling to explore beyond the starting chamber of the maze. By excluding this time of heightened arousal, this measure highlighted that chicks are significantly more motivated to explore the maze to approach the bobwhite maternal call than to explore the maze to avoid the aversive novel tone. This measure generally corrected more for the subjects in 
the Avoidance condition, as they had a significantly longer latency to emerge from the starting chamber of the maze than the chicks in the Approach condition (Table 5).

The results of this study have important implications for the development of explorative tendencies in young animals and infants, as well as across the lifespan. The present study also has important methodological implications for researchers that focus on pretrial manipulations without controlling for stimuli present during testing that may be affecting level of emotional reactivity and exploration motivation.

My study can serve as a basis for future research on emotional reactivity and its effects, not only on exploration, but also on such functions as cognition, memory, and learning. This study can also be expanded by comparing chicks of different developmental ages or different animal species altogether to determine the extent to which the present findings generalize. It would also be beneficial to conduct similar studies using more ecologically valid testing apparatuses and aversive auditory stimuli to determine if the present results would generalize readily to animals in their natural habitats. Furthermore, it is important to keep in mind that although the stimuli present at any given time are critical components for influencing level of emotional reactivity and exploration motivation, there are numerous factors present throughout early development that are likely to influence these phenomena. 


\section{REFERENCES}

Boissy, A. (1995). Fear and fearfulness in animals. Quarterly Review of Biology, 70, 165191.

Boissy, A. \& Bouissou, M. F. (1995). Assessment of individual differences in behavioural reactions of heifers exposed to various fear eliciting situations. Applied Animal Behaviour Science, 46, 17-31.

Bolhuis, J. E., Schouten, W. G. P., de Leeuw, J. A., Schrama, J. W., \& Wiegant, V. M. (2004). Individual coping characteristics, rearing conditions and behavioural flexibility in pigs. Behavioral Brain Research, 152, 351-360.

Elliot, A. J. (1999). Approach and avoidance motivation and achievement goals. Educational Psychologist, 34, 169-189.

Elliot, A. J. (2006) The hierarchical model of approach-avoidance motivation. Motivation Emotion, 30, 111-116

Elliot, A. J. \& Covington, M. V. (2001). Approach and avoidance motivation. Educational Psychology Review, 14, 73-92.

Elliot, A. J., \& Thrash, T. M. (2002). Approach-avoidance motivation in personality: Approach and avoidance temperaments and goals. Journal of Personality and Social Psychology, 82, 804-818.

Freire, R., Cheng, H. W. \& Nicol, C. J. (2004). Development of spatial memory in occlusion-experienced domestic chicks. Animal Behaviour, 67, 141-150.

Goddard, M. E. \& Beilharz, R. G. (1984). A factor analysis of fearfulness in potential guide dogs. Applied Animal Behaviour Science, 12, 253-265.

Gray, L. (1990). Activity level and auditory responsiveness in neonatal chickens. Developmental Psychobiology, 23, 297-308.

Heaton, M. B., Miller, D. B., \& Goodwin, D. G. (1978). Species-specific auditory discrimination in bobwhite quail neonates. Developmental Psychobiology, 11, 1321 .

Hernsworth, P. H., Barnett, J. L., \& Jones, R. B. (1993). Situational factors that influence the level of fear of humans by laying hens. Applied Animal Behaviour Science, 36, 297-210. 
Imada, H. (1970). Amount of open-field defecation, home cage defecation and food and water intake in maudsley reactive and nonreactive strains of rats. The Annual of Animal Psychology, 20, 1-6.

Jones, R. B. (1988). Repeatability of fear ranks among adult laying hens. Applied Animal Behaviour Science, 19, 297-304.

Jones, R. B. (2002). Role of comparative psychology in the development of effective environmental enrichment: Strategies to improve poultry welfare. International Journal of Comparative Psychology, 15, 77-106.

Lazic, M., Schneider, S. M., \& Lickliter, R. (2007). Enriched rearing facilitates spatial exploration in Northern bobwhite (Colinus virginianus) neonates. Developmental Psychobiology, 49, 548-551.

Lickliter, R. (1989). Species-specific auditory preference of bobwhite quail chicks (Colinus virginianus) is altered by social interaction with siblings. Journal of Comparative Psychology, 103 (3), 221-226.

Lickliter, R, Bahrick, L. E., \& Honeycutt, H. (2002). Intersensory redundancy facilitates prenatal perceptual learning in bobwhite quail embryos. Developmental Psychology, 38, 15-23.

Lickliter, R., Bahrick, L. E., \& Honeycutt, H. (2004). Intersensory redundancy enhances memory in bobwhite quail embryos. Infancy, 5, 253-269.

Lickliter, R., Dyer, A., \& McBride, T. (1993). Perceptual consequences of early social experience in precocial birds. Behavioural Processes, 30, 185-200.

Lyons, D. M. (1989). Individual differences in temperament of dairy goats and the inhibition of milk ejection. Applied Animal Behaviour Science, 22, 269-282.

Manteca, X. \& Deag, J. M. (1993). Individual differences in temperament of domestic animals: A review of methodology. Animal Welfare, 2, 247-268.

Mench, J. A. (1992). The welfare of poultry in modern production systems. Poultry Science Review, 4, 107-128.

Miller, K. A., Garner, J. P., \& Mench, J. A. (2005). The test-retest reliability of four behavioural tests of fearfulness for quail: A critical evaluation. Applied Animal Behaviour Science, 92, 113-127.

Miller, K. A. \& Mench, J. A. (2005). The differential use and effects of four types of environmental enrichment on the activity budgets, fearfulness, and social 
proximity preference of Japanese quail. Applied Animal Behaviour Science, 95, 169-187.

Miller, K. A., Garner, J. P., \& Mench, J. A. (2006). Is fearfulness a stable and consistent trait that is measurable using behavioral tests? Animal Behaviour, 71, 1323-1334.

Mills, A. \& Faure, J. M. (1986). The estimation of fear in domestic quail: correlations between various methods and measures. Biology of Behavior, 11, 235-243.

Mills, A. D. \& Faure. J. M. (2000). Ease of capture in lines of Japanese quail (Coturnix japonica) subjected to contrasting selection for fear or sociability. Applied Animal Behaviour Science, 69, 125-134.

Molina-Hernandez, M., Tellez-Canatara, P., \& Perez-Garcia, J. (2001). Isolation rearing induced fear-like behavior without affecting learning abilities of Wistar rats. Progress in Neuro-Psychopharmacology and Biological Psychiatry, 25, 11111123.

Mowrer, O. H. (1939). A stimulus-response analysis of anxiety and its role as a reinforcing agent. Psychological Review, 46, 553-565.

Ortet, G., \& Ibanez, M. I. (1999). Open-field exploration and emotional reactivity in mice. Psichotherma, 11, 75-81.

Sackett, G. P. (1965). Effects of rearing conditions upon the behavior of rhesus monkeys. Child Development, 36, 855-868.

Schneirla, T. C. (1959). An evolutionary and developmental theory of biphastic processes underlying approach and withdrawal. In Jones, M. R. (ed.) Nebraska symposium on motivation. Vol. 7. Lincoln: University of Nebraska Press, pp. 1-42.

Schneirla, T. C. (1965). Aspects of stimulation and organization in approach/withdrawal processes underlying vertebrate behavioral development. In D. Lehrman, R. Hinde, \& E. Shaw (eds.) Advances in the study of behavior. Vol. 1. New York: Academic Press, pp. 1-74.

van der Staay, F. J., Schuurman, T., van Reenen, C. G., \& Korte, S. M. (2009). Emotional reactivity and cognitive performance in aversively motivated tasks: A comparison between four rat strains. Behavioral and Brain Functions, 5:50.

van Hoek, C. S. \& King, C. E. (1997). Causation and influence of environmental enrichment on feather pecking of the crimson-bellied conure (Pyrrhura perlata perlata). Zoo Biology, 16, 161-172. 
Vandenheede, M. \& Bouissou, M. F. (1998). Effects of an enriched environment on subsequent fear reactions of lambs and ewes. Developmental Psychobiology, 33, $33-45$. 